\title{
Tubulocystic Renal Cell Carcinoma
}

National Cancer Institute

\section{Source}

National Cancer Institute. Tubulocystic Renal Cell Carcinoma. NCI Thesaurus. Code C126303.

A rare, cytologically high grade carcinoma that arises from the renal parenchyma. It is characterized by the presence of prominently dilated cystic spaces in a bland, fibrotic stroma. The lining cells have large irregular nuclei with prominent nucleoli and abundant eosinophilic cytoplasm. Necrosis and mitotic figures are not present. It rarely metastasizes. 